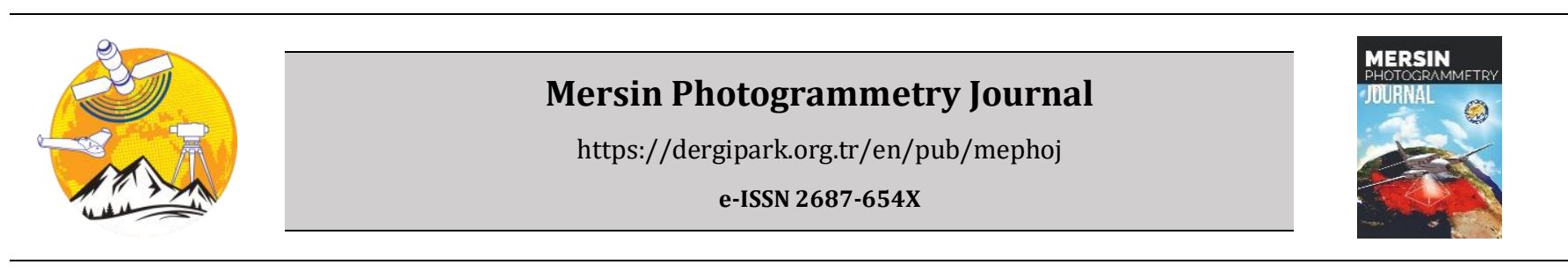

\title{
Photogrammetric analysis of multispectral and thermal close-range images
}

\author{
Özgün Akçay*1] \\ ${ }_{1}^{1}$ Canakkale Onsekiz Mart University, Faculty of Engineering, Department of Geomatics Engineering, Canakkale, Turkey
}

\author{
Keywords \\ Photogrammetry \\ Remote sensing \\ Multispectral images \\ Thermal images \\ Close-range
}

\begin{abstract}
Sensors capable of multispectral and thermal imaging beyond visible bands offer many analysis possibilities for environmental monitoring. Different sensor images constitute an important source of information especially in the fields of agriculture, forestry, geology and energy. Photogrammetric studies have been affected by this development in recent years and have been used in the production of multispectral and thermal models besides the RGB model. However, due to geometric and radiometric resolution differences, it is difficult to combine or evaluate models produced from different types of sensors. In this study, the three-dimensional test field images obtained with RGB, multispectral and thermal sensors were oriented and modeled photogrammetrically. The accuracies of the control points on the produced models were compared and discussed. When the results are examined, control point accuracy was obtained as almost similar as in the RGB model after the orientation based on automatic feature matching. Automatic feature detection and matching in thermal images were not robustly produced due to low geometric resolution. For this reason, manual measurements were performed in thermal images, and the photogrammetric orientation and adjustment process was done accordingly. The fused evaluation approach considering RGB, multispectral and thermal images in one photogrammetric model was also implemented and discussed.
\end{abstract}

\section{INTRODUCTION}

Recently, multi-sensor modeling and analyzes have been carried out with terrestrial and UAV-based closerange photogrammetry. Although multispectral and thermal lightweight cameras are relatively low resolution compared to RGB cameras, photogrammetric products three-dimensional models and orthophotos have been considered for monitoring and inspection in many areas such as forestry, agriculture and archaeology.

Studies using multispectral camera images have been involved in photogrammetric processes, especially in agriculture and forestry. Nebiker et al. (2008) presented investigations using low-weight and low-cost multispectral sensors in combination with mini and micro UAVs for remote sensing applications in agricultural test fields. A fully automated digital multispeetral and high resolution image acquisition and 3D-processing system was proposed by Wewel et al., (2014). The multispectral data were processed with a photogrammetric pipeline to create triband orthoimages to extract some Vegetation Indices (VI) such as the Normalized Difference Vegetation Index (NDVI), the Green Normalized Difference Vegetation Index (GNDVI), and the Soil Adjusted Vegetation Index (SAVI), examining the vegetation vigor for each crop (Candiago et al., 2015). Saura et al. (2019) also analysed a vineyard with UAV based multispectral imagery and produced the Digital Elevation Model and NDVI to collect information about the agricultural production such as moisture and biomass density. Minařík and Langhammer (2016) proposed a methodology for assessment of spatial and qualitative aspects of forest disturbance based on the multispectral sensor Tetracam camera with the UAV photogrammetry. Sankey et al. (2021) demonstrated that a model incorporating the fusion of UAV multispectral and structure-from-motion photogrammetry classifies plant functional types and bare soil cover with an overall accuracy of $95 \%$ in rangelands degraded by shrub encroachment and disturbed by fire. Xu et al. (2020) acquired high spatial resolution multispectral and RGB imagery over a 
subtropical natural forest in southwest China using a fixed-wing UAV system and derived 3D point cloud.

On the other hand, photogrammetric studies which consider both RGB and thermal images are found in the literature. Ribeiro-Gomes et al. (2017) evaluated the use of the Wallis filter for improving the quality of the thermal photogrammetry process using structure from motion software. Despite the low resolution of the thermal imagery compared to RGB imagery, forest structural elements were extracted using both point clouds (Webster et al. 2018). Van der Sluijs et al. (2018) revealed the morphology and daily to annual dynamics of thaw-driven mass wasting phenomenon using photogrammetric terrain models and orthomosaic time series. Zefri et al. (2018) studied about the use of thermal and visual imagery taken by UAV in the inspection of photovoltaic installations. Biass et al. (2019) provided detail in characterizing the emplacement of a compound pāhoehoe lava flow using SfM photogrammetry techniques to visible and thermal data sets. Zumr et al. (2020) utilized a combination of electrical resistivity tomography, close range photogrammetry, and unmanned aerial vehicle (UAV) thermal imaging techniques to detect specific superficial and internal structures of a historical earth-filled dam. A low-cost method was proposed to monitor Stromboli, a volcano in the Aeolian Islands of Italy using a unique 3D thermal photogrammetric modelling workflow. The data acquisition and processing part of this workflow has been tested on Stromboli, a volcano in the Aeolian Islands of Italy (Wakeford et al., 2020).

Studies in which multispectral and thermal threedimensional photogrammetric models and their analysis are performed together can also be found in the literature. Erenoglu et al. (2017) developed a novel methodology extracting and distinguishing material features from UAS-based multi-sensor data photogrammetry for the cultural heritages. Edelman (2018) explored the feasibility to obtain visible, infrared, hyperspectral and thermal 3D registrations of simulated crime scenes using photogrammetry for use in forensic practice. Raeva et al. (2019) carried out unmanned flights with a fixed-wing platform with two different sensors - multispectral and thermal in order to examine two main crops cultivated area. Turner et al. (2020) investigated geological discontinuities in hard rock masses using UAV-mounted thermal and multispectral cameras. Matese and Di Gennaro (2018) described the implementation of a multisensor UAV system capable of flying with three sensors simultaneously to present performances in the characterization of spatial variability in terms of vegetative vigor, water stress and missing plant detection.

When the studies in the literature are examined, it is seen that the geometric accuracy analysis of the photogrammetric models of different sensor images has not been considered concurrently. The scope of this study is to compare the photogrammetric threedimensional point accuracies, point clouds and polygons produced using test area images of sensors having different spectral ranges and geometric resolutions. In this study, the three-dimensional test field images obtained with RGB, multispectral and thermal sensors were oriented photogrammetrically and the accuracies of the control points on the obtained models were compared. In particular, the effects of sensors on photogrammetry as a result of modeling with multispectral and thermal images were discussed.

\section{METHODOLOGY}

All photogrammetric experiments were carried out on the three-dimensional test field as shown in Fig. 1. The predefined coded targets of Photomodeler and Agisoft Metashape software have been fixed on the test area in dimensions of $85 \mathrm{~cm}$ x $85 \mathrm{~cm}$ x $20 \mathrm{~cm}$. Providing depth variations in control points produces more reliable results during interior and exterior camera orientations (Fraser, 2013). In order to have higher accuracy, the ratio of test field depth to image shooting distance was determined as 0.25 , while a nadir viewing angle was preferred.

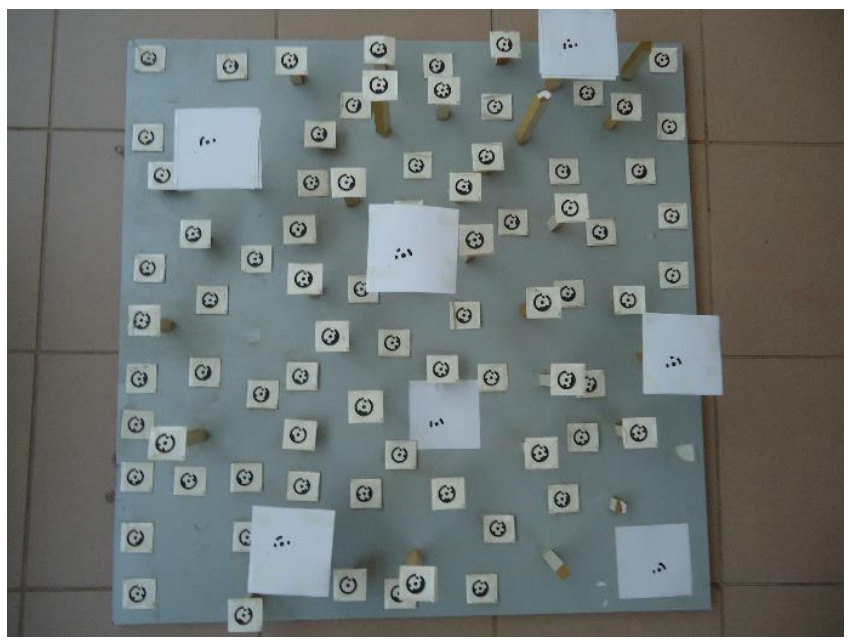

Figure 1. Three dimensional test field

As indicated in the workflow in Fig. 2, firstly, the coordinates of the control points were calculated on the test field using Sony RGB photogrammetric model. Then, photogrammetric models were created by taking test field images with Mapir Survey3 OCN multispectral and Optris PI 450 thermal cameras.

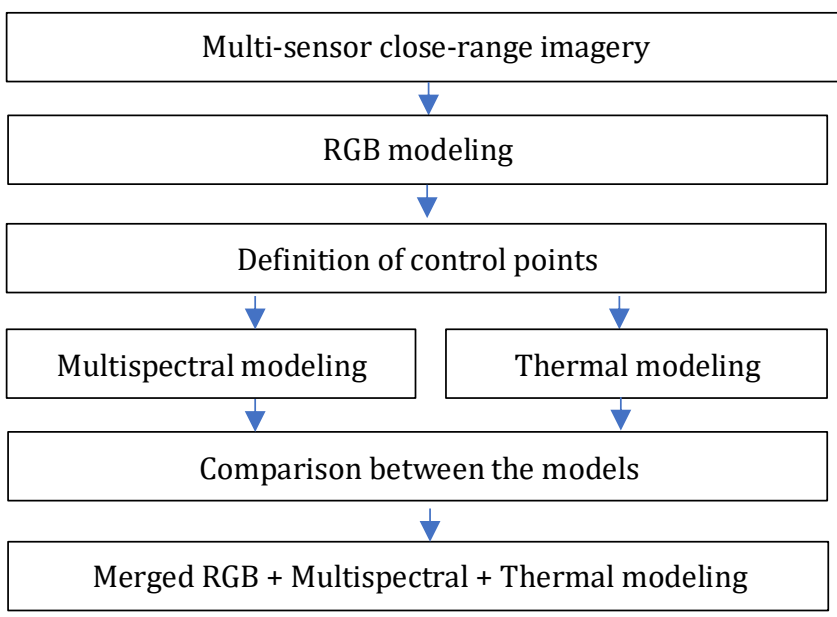

Figure 2. Workflow for photogrammetric analysis 
The features of the cameras used in the study are shown in Table 1. Although the geometric resolutions of RGB cameras and multispectral cameras are close to each other, it is seen that the resolution of the thermal camera is quite low compared to the others. In the results section, the accuracies produced from photogrammetric models are presented. Finally, three photogrammetric models obtained using different sensor have been optimized and merged.

Table 1. Sensor specifications

\begin{tabular}{llll}
\hline Name & Bands & $\begin{array}{l}\text { Resolution } \\
\text { (pixel) }\end{array}$ & $\begin{array}{l}\text { Spectral } \\
\text { wavelength } \\
\text { range (nm) }\end{array}$ \\
\hline Sony & Red Green Blue & $4320 \times 3240$ & $400-700$ \\
Mapir & Orange Cyan NIR & $4000 \times 3000$ & $450-900$ \\
Optris & Thermal & $382 \times 288$ & $(8-14) \times 10^{3}$ \\
\hline
\end{tabular}

\subsection{Photogrammetric Control Points}

The coordinates of the control points were also produced by the photogrammetric method. By using 10 images taken with the RGB camera, 75 coded targets were automatically measured with Photomodeler, then the orientation and bundle adjustment processes were completed. Modern bundle adjustment assessments and software solutions were discussed in Murtiyoso et al. (2018). Measurements of 7 coded targets (CP1, CP2, CP4, CP6, CP8, CP10 and CP12) belonging to Agisoft software were performed by manual photogrammetric method and model coordinates were generated. After the distance between the targets P1 and P8 was determined with a precision ruler, the model coordinate system was transformed into the local coordinate system defined in the metric system. In Agisoft software, 10 image orientations were re-processed with coded targets, and $\mathrm{X}, \mathrm{Y}, \mathrm{Z}$ coordinates of control points and their errors were defined as seen in Table 2. The table shows that the point location accuracies are between about $0.9 \mathrm{~mm}$ and $1.3 \mathrm{~mm}$, while the elevation of the control points varies between $18.6 \mathrm{~cm}$.

Table 2. Control points in the local coordinate system

\begin{tabular}{lllll}
\hline No & $\mathrm{X}(\mathrm{m})$ & $\mathrm{Y}(\mathrm{m})$ & $\mathrm{Z}(\mathrm{m})$ & Error $(\mathrm{m})$ \\
\hline CP1 & 0.3750 & -0.2895 & -0.8057 & 0.0009 \\
CP2 & 0.3557 & 0.0122 & -0.6887 & 0.0013 \\
CP4 & 0.0197 & 0.1448 & -0.6212 & 0.0013 \\
CP6 & -0.1406 & -0.2054 & -0.6868 & 0.0011 \\
CP8 & 0.0570 & -0.0760 & -0.8068 & 0.0012 \\
CP10 & 0.2448 & 0.4398 & -0.7356 & 0.0012 \\
CP12 & -0.2293 & 0.3003 & -0.6830 & 0.0013 \\
\hline
\end{tabular}

\subsection{Multispectral and Thermal Models}

First, radiometric calibration of the multispectral camera images was performed in order to build quantitative spectral and spatial representations of the test field (Guo et al., 2019). Reflectance calibration target was used during radiometric correction of multispectral images. The pixel values of the captured target image are compared with the known reflectance values of the targets. Mapir calibration software then transform the pixel values and thus calibrate the test field images. The 12 images obtained as a result of the calibration were automatically oriented and optimized by the coded targets in Agisoft software. In addition, dense point clouds were produced (de Lima et al., 2021) using oriented multispectral images as depicted in Fig. 3. It was observed that multispectral imagery enables orthophotos production as well as digital surface model.

Since 8 - $14 \mu \mathrm{m}$ spectral range of thermal camera images is different than RGB and multispectral sensor images, it was not possible to automatically measure the coded targets in the Agisoft Metashape software. Due to noise and radiometric distortions on the thermal images, automatic feature detection and matching was not sufficient for orientation.

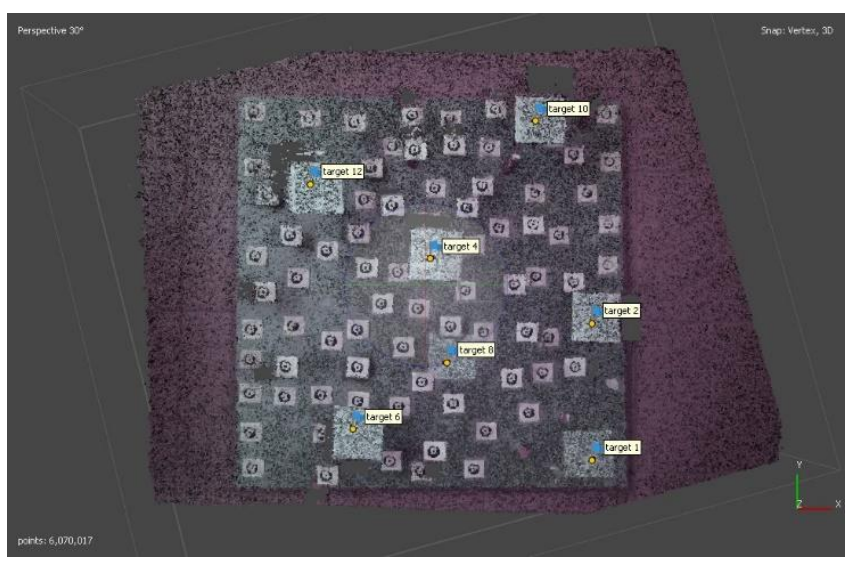

Figure 3. Multispectral dense cloud

Thermal image contents are also affected by different view angles of the camera and each object emits thermal radiation in a certain manner, as it changes its geometry (Athanasopoulos \& Siakavellas. 2017). Fig. 4 shows the views of the same features in different orientated thermal images. 7 coded targets and 11 other targets were manually measured on 13 thermal images. Correct emissivity values could provide valuable information concerning the interpretation of thermal images obtained from thermographic surveys (Avdelidis \& Moropoulou, 2003). The selectivity of some targets for manual measurements has been increased by metal coins. As the metal materials, have lower emissivity values, targets become more determined for image measurements in the thermal images.
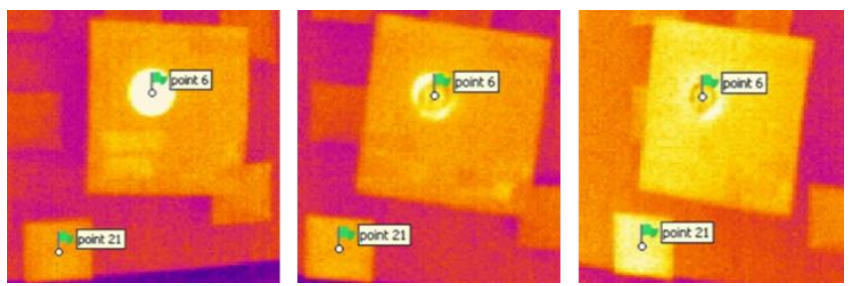

Figure 4. Same features on different thermal images

Beside the disadvantage of low geometric resolution, Fig. 5 explicitly depicts high distortion, low contrast and blur effect that reduce image quality dramatically (Mello Román et al., 2019). Therefore, the loss of details, which is an unavoidable result for thermal imagery, causes obstacles during three- 
dimensional photogrammetric modeling process. Photogrammetric measurement points can also be seen in a thermal image in Fig. 5.

Another obstacle in thermal photogrammetry is outer heat sources occurred in scene during image taking. This situation leads to global noise effects in image frame. As seen in Fig. 5, at the right side of the image, purple, which represents lower emissivity whereas the top left corner brighter pixels, which represents higher emissivity due to external heat source.

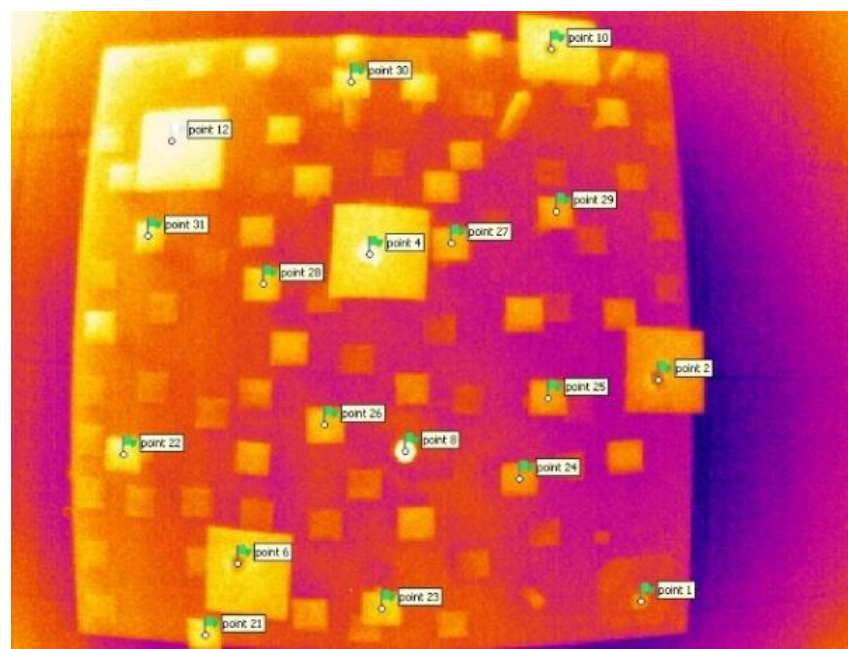

Figure 5. A thermal image and measurement points

By measuring manually a total of 18 common points on the images, orientation and optimization were implemented successfully (Fig. 5). Dense point clouds could not be produced due to insufficient automatic matching based on the thermal image. A dense surface can only be created by an intensive manual measurement based on the oriented thermal model; as it is beyond the scope of this study, sparse thermal point cloud was used during point cloud comparisons.

\subsection{Fused Photogrammetric Model}

Three photogrammetric models using RGB, multispectral and thermal images were reconsidered for an unified orientation and bundle adjustment in order to produce a fusion model. The purpose of the fusion model is to optimize separate photogrammetric models all together. It enables compare different spectral values of any point or location. The fusion model consists of 10 RGB images, 12 multispectral images and 13 thermal images were carried out using 54 manual and coded marking points, and 10459 automatch tie points. Successively, dense point cloud having 6179491 points was obtained as depicted in Fig. 6. Points of dense point cloud were colored using three different sensor images, although points were mostly colored with RGB and multispectral data.

On the other hand, the mosaic of orthophotos, which contains more thermal patches, was automatically produced as shown in Fig 7. The resolution of the orthomosaics was calculated as 0.312 $\mathrm{mm} /$ pix. Fused model imply that the thermal images might be utilized during orthophotos production, even if single thermal model is not able to accomplish photogrammetric products such as dense point cloud, surface model and orthophotos.

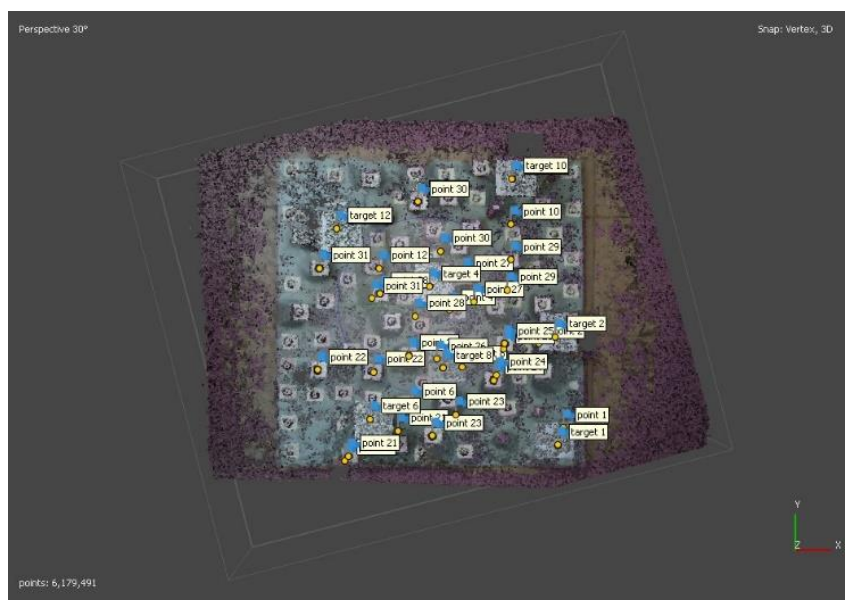

Figure 6. Dense cloud of fusion model

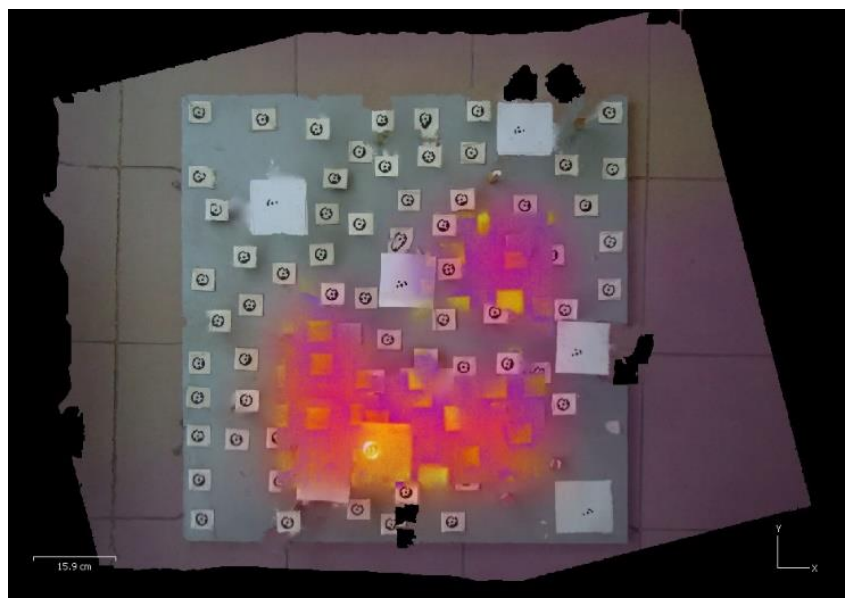

Figure 7. Orthomosaic of fusion model

\section{RESULTS AND DISCUSSIONS}

Photogrammetric models are evaluated in three subsections; first subsection regards control point measurement accuracies, second one is considered as evaluation of point cloud products whereas third one compares polygonal shapes.

\subsection{Control Points Accuracies}

When pixel errors were examined in Table 3 and Fig. 7, it is seen that photogrammetric measurement accuracies are similar in all image types. In other words, the accuracy of manual measurements made in thermal and automatic measurements in RGB and multispectral images produced similar results. In some control points, it is also observed that manual thermal measurements are better than automatic multispectral measurements. As a result, the lowest projection errors accomplished with RGB photogrammetric model.

However, when metric accuracies are analyzed in Table 4 and Fig. 8, it is seen that RGB and multispectral results differ significantly from thermal results. Although RGB stands out in terms of accuracy, the results obtained from multispectral images are also very consistent. This is due to the fact that one pixel size of 
the thermal camera sensor is larger than other sensors. Table 5 describes differences of common control points between RGB model and multispectral models, while Table 6 explains differences between RGB and Thermal models. Results of coordinate differences prove that RGB and multispectral models are quite coherent. On the other hand, CP2, CP4 and CP12 are observed as the most biased points between the discussed models. The comparison of RGB and Thermal model indicate that CP2 and CP6 have more variance than other control points.

Table 3. Errors of control points obtained from different imagery models in pixel unit

\begin{tabular}{llll}
\hline No & RGB & Multispectral & Thermal \\
\hline CP1 & 0.231 & 0.343 & 0.523 \\
CP2 & 0.276 & 0.476 & 0.290 \\
CP4 & 0.215 & 0.293 & 0.278 \\
CP6 & 0.252 & 0.558 & 0.290 \\
CP8 & 0.192 & 0.144 & 0.334 \\
CP10 & 0.174 & 0.299 & 0.337 \\
CP12 & 0.289 & 0.598 & 0.386 \\
\hline
\end{tabular}

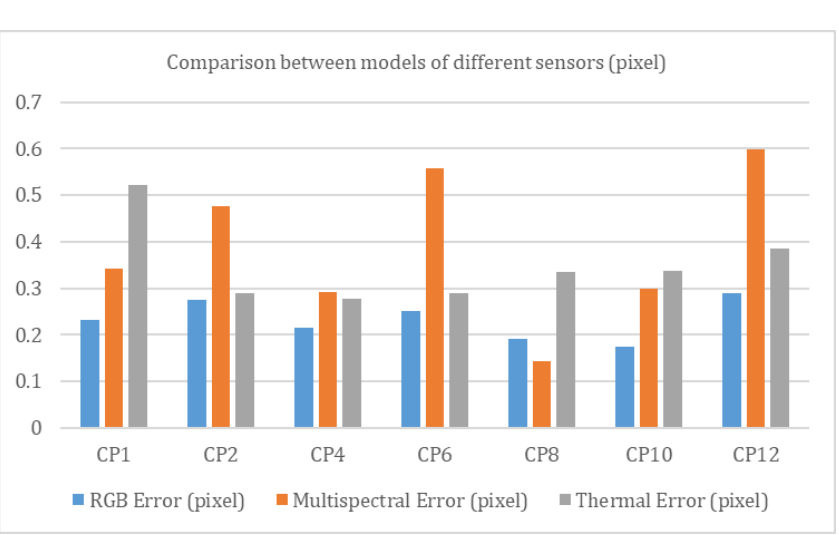

Figure 7. Comparison between in pixel unit

Table 4. Errors of control points in millimeter unit

\begin{tabular}{llll}
\hline No & RGB & Multispectral & Thermal \\
\hline CP1 & 0.9 & 1.7 & 2.9 \\
CP2 & 1.3 & 1.6 & 3.5 \\
CP4 & 1.3 & 1.1 & 3.1 \\
CP6 & 1.1 & 0.7 & 4.8 \\
CP8 & 1.2 & 1.2 & 1.5 \\
CP10 & 1.2 & 1.3 & 1.8 \\
CP12 & 1.3 & 1.5 & 1.7 \\
\hline
\end{tabular}

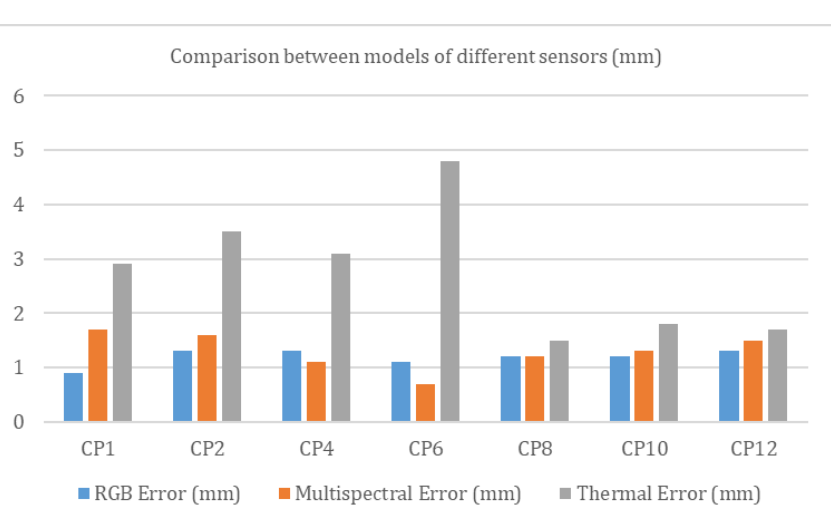

Figure 8. Comparison between errors in millimeter unit
Table 5. Differences of common control points between RGB model and multispectral models

\begin{tabular}{llll}
\hline No & $\Delta \mathrm{X}(\mathrm{mm})$ & $\Delta \mathrm{Y}(\mathrm{mm})$ & $\Delta \mathrm{Z}(\mathrm{mm})$ \\
\hline CP1 & +0.3 & -0.7 & -0.4 \\
CP2 & -0.9 & +0.9 & 0.0 \\
CP4 & +0.6 & +1.0 & -0.6 \\
CP6 & +0.7 & -0.8 & +0.1 \\
CP8 & -0.6 & -1.0 & +0.1 \\
CP10 & -0.8 & -0.5 & +0.8 \\
CP12 & +0.7 & +1.1 & -0.2 \\
\hline
\end{tabular}

In the study, as mentioned in methodology, the fusion model was also implemented considering RGB, multispectral and thermal images in one project. However, image measurements of control points in thermal image substantially diverge from other image measurements in the fusion model. Pixel errors in thermal images vary between 0.914 and 9.476 while errors in three-dimensional coordinate system differ between $11 \mathrm{~cm}$ and $27 \mathrm{~cm}$. The reason for the high deviations is that automatic coded target measurements cannot be performed in thermal images. Besides, tie points which detected using SIFT algorithm were also presented low matching accuracy among thermal images. Deviations of thermal image points from RGB image measurements can be observed in Fig. 9.

Table 6. Differences of common control points between RGB model and thermal models

\begin{tabular}{llll}
\hline No & $\Delta \mathrm{X}(\mathrm{mm})$ & $\Delta \mathrm{Y}(\mathrm{mm})$ & $\Delta \mathrm{Z}(\mathrm{mm})$ \\
\hline CP1 & +1.5 & +0.6 & +2.0 \\
CP2 & +1.4 & +2.1 & -2.2 \\
CP4 & +0.1 & -0.3 & +2.2 \\
CP6 & -2.6 & +0.9 & -2.9 \\
CP8 & -1.1 & -2.4 & +0.6 \\
CP10 & -0.9 & -1.2 & -0.8 \\
CP12 & +1.6 & +0.4 & +1.1 \\
\hline
\end{tabular}

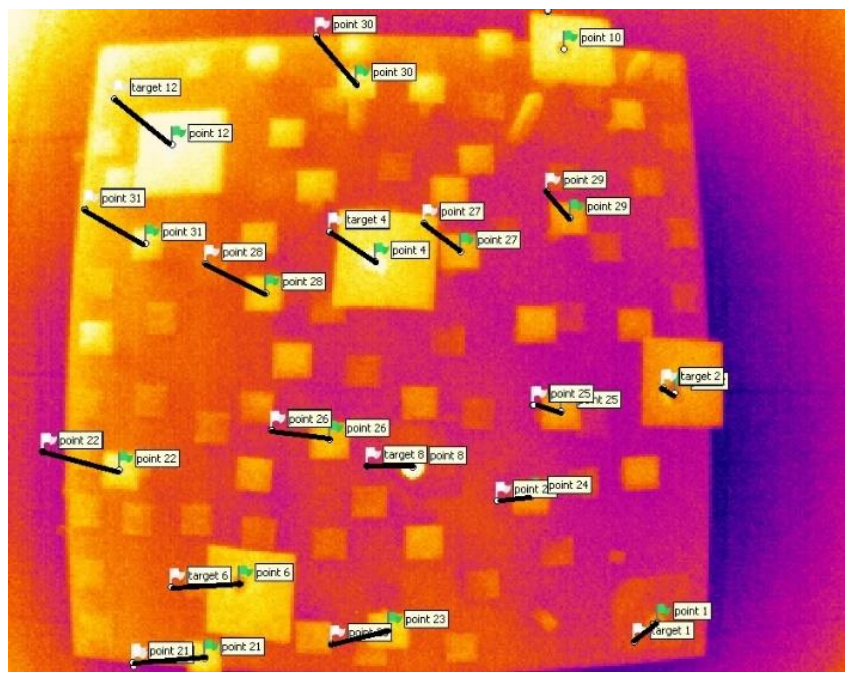

Figure 9. Deviations of thermal image points

\subsection{Comparison of Point Clouds}

Dense point clouds were produced from RGB and multispectral model as shown in Fig 10 and 11. However, thermal photogrammetric model was able to spare point cloud that represent test field (Fig. 12). The 
dense point clouds were utilized for digital surface model production, which is essential for obtaining orthophotos. Thermal model was oriented and optimized, yet, digital surface model and orthophotos could not be accomplished from sparse point cloud. The only way getting thermal orthophotos is to use the fused model, however, it arises high measurement error issue for thermal images at that time. Another solution for thermal orthophotos production is increasing manual corresponding image point measurements, although it is a time consuming method in photogrammetry. Therefore sparse point cloud can be intensified so as to provide more discrete three-dimensional points for obtaining DSM models such as delaunay triangulation and inverse distance weighting (Wang et al., 2020).

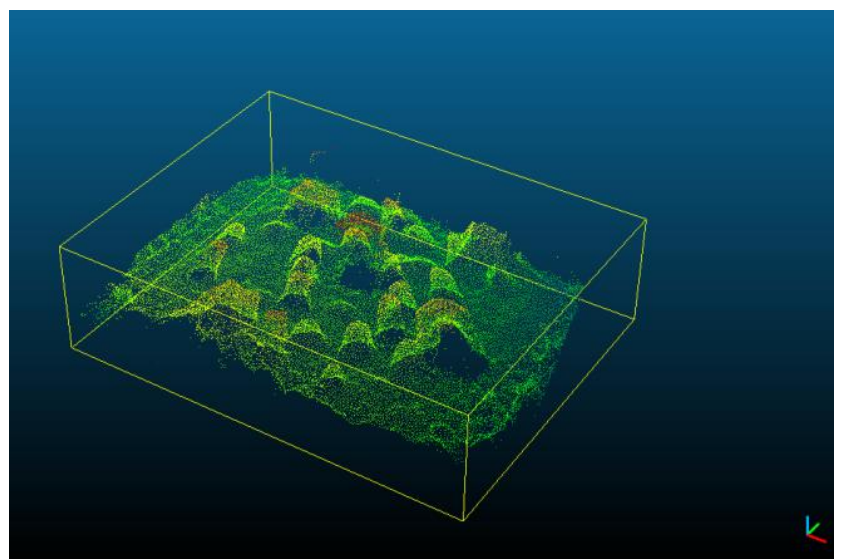

Figure 10. Dense point cloud of RGB model

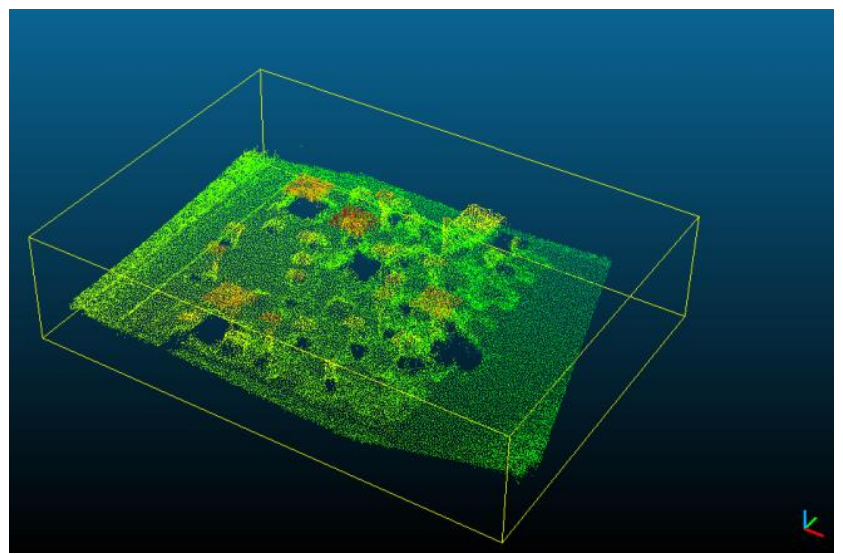

Figure 11. Dense point cloud of multispectral model

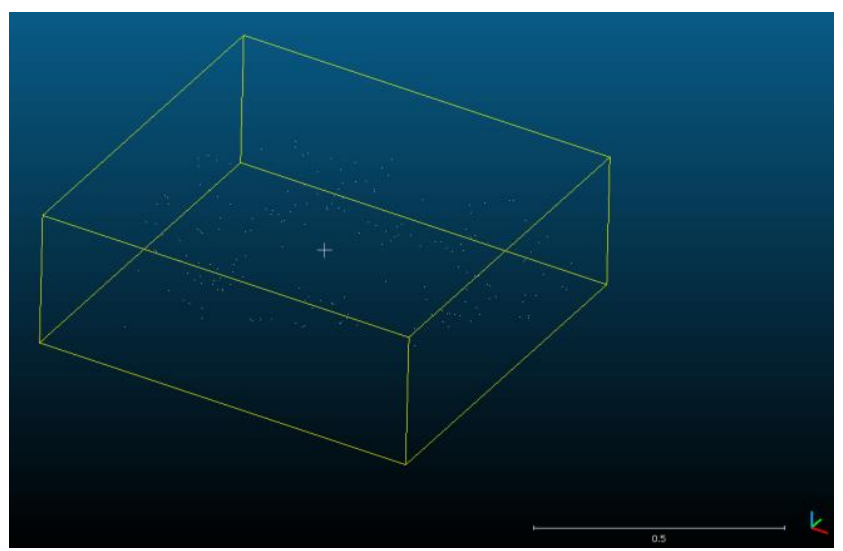

Figure 12. Sparse point cloud of thermal model
Number of point of RGB, multispectral and thermal clouds are 109489,6069889 and 185 , respectively. It is also seen that RGB and multispectral points' heights tend to similar distribution when their histogram analyzed (Fig. 13 and 14). In contrast to the dense point clouds, the distribution of the heights of sparse thermal points presents low sampling rates to represent the test field (Fig. 15). Consequently, thermal imagery using 382 x 288 resolution camera leads significant challenges as concerning multi sensor photogrammetric modelling.

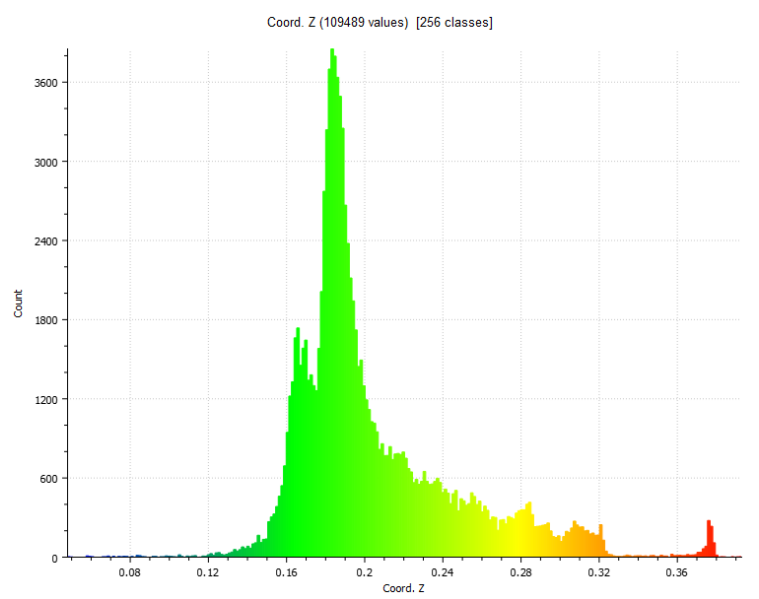

Figure 13. The distribution of $\mathrm{Z}$ coordinates of $\mathrm{RGB}$ dense point cloud

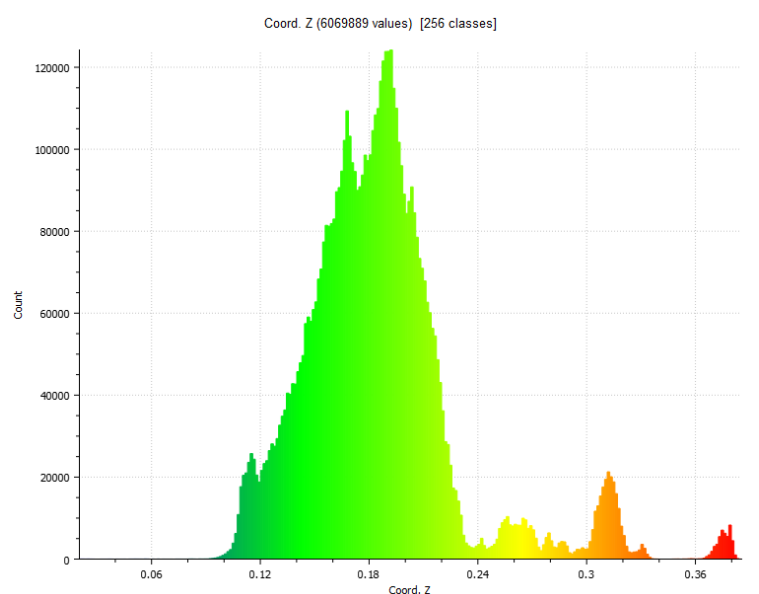

Figure 14. The distribution of coordinates $\mathrm{Z}$ of multispectral dense point cloud

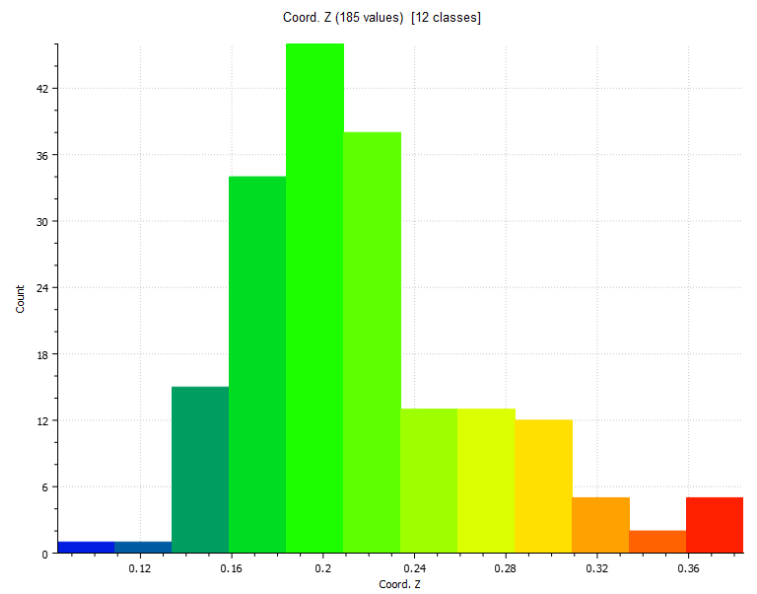

Figure 15. The distribution of coordinates $\mathrm{Z}$ of thermal sparse point cloud 
The section (Section 1) and its four cross sections (CS1, CS2, CS3, and CS4) (Fig. 16) was determined on the test field and, then, $\mathrm{Z}$ values extracted from RGB and multispectral point clouds. In Fig. 17, Z coordinate values of RGB and multispectral points are represented as green and red lines, respectively.

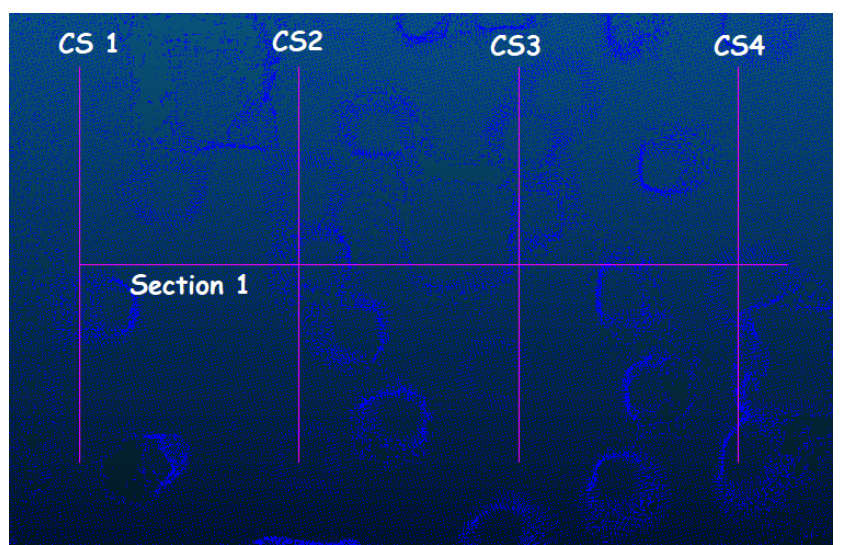

Figure 16. Section 1 and its four cross sections CS1, CS2, CS3 and CS4
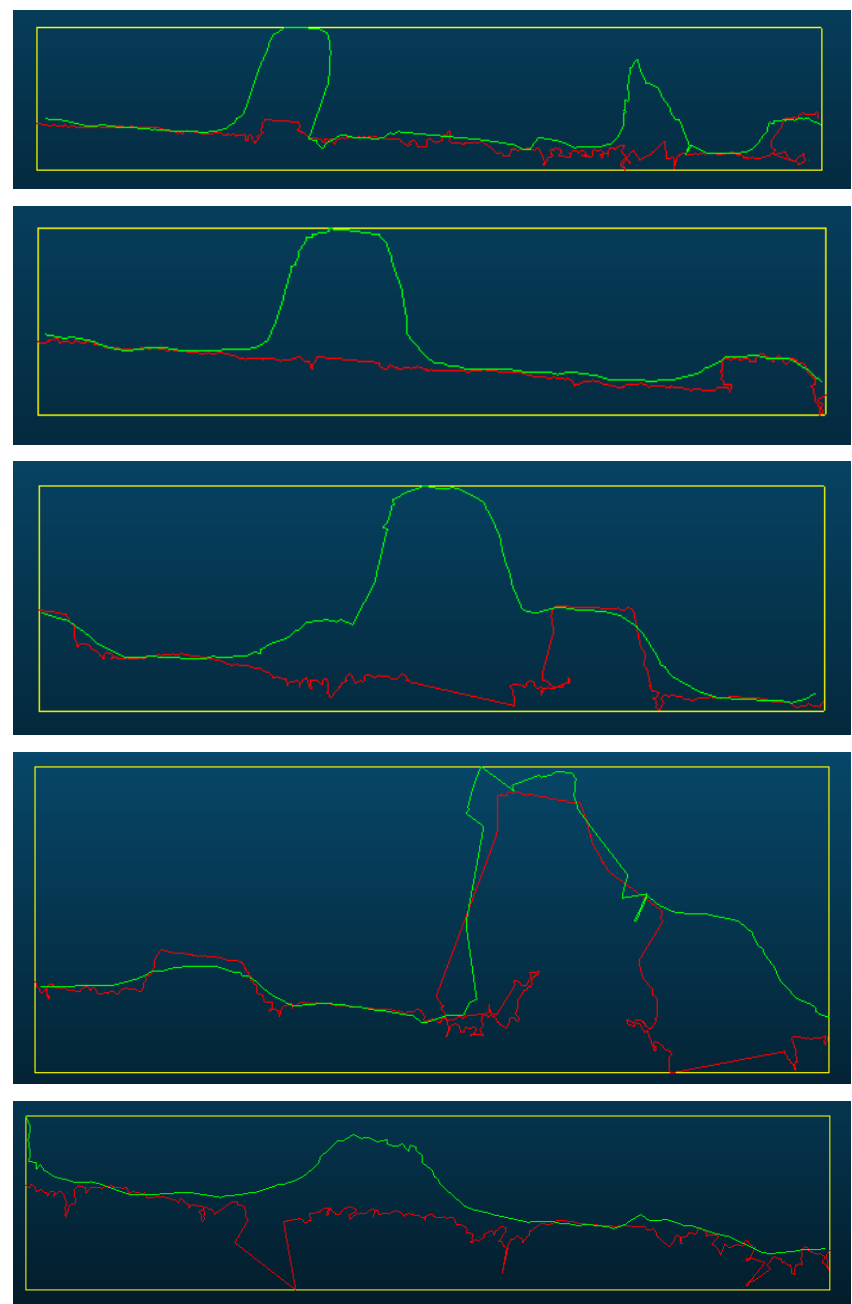

Figure 17. Z coordinate values of Section 1, CS1, CS2, CS3 and CS4 from RGB and multispectral point clouds are presented from top to down

\subsection{Comparison of Extracted Polygons}

Photogrammetry is also one of the important geometric data sources such as lines and polygons for geospatial applications. Therefore, in the scope of this study, polygons were created manually from photogrammetric models and their perimeters and areas were compared (Table 7). Polygons were obtained from 8 photogrammetric point vertices (CP1, CP2, CP10, CP12, CP21, CP22, CP30, CP31). Results show that all models presents significant metric accuracy as perimeter differences between polygons of RGB and the other models are $2 \mathrm{~mm}$ and $14 \mathrm{~mm}$. Areas of polygons also validate coherence among multi-sensor models.

Table 7. Calculated measures of polygons

\begin{tabular}{lll}
\hline Model type & Perimeter $(\mathrm{mm})$ & Area $\left(\mathrm{cm}^{2}\right)$ \\
\hline Polygon-RGB & 2449 & 3997 \\
Polygon-Multispectral & 2451 & 3995 \\
Polygon-Thermal & 2463 & 4072 \\
\hline
\end{tabular}

\section{CONCLUSIONS}

In photogrammetry, besides three-dimensional RGB models, multispectral and thermal models were successfully produced. In particular, radiometric corrections on multispectral and thermal images are important in terms of geometric positioning accuracy. Due to the difficulties in automatic processing of thermal images, mandatory manual point measurement causes a time-consuming model production and accuracy losses. The low resolution of thermal images makes it difficult to combine and analyze them with RGB and multispectral models. In the future, new thermal photogrammetric studies which deal data fusion with RGB and multispectral camera images should be carried out to increase the resolution of thermal models. The fused photogrammetric model was produced successfully, however specific approaches need to be developed in order to increase model performance and product quality.

\section{Conflicts of interest:}

The authors declare no conflicts of interest.

\section{REFERENCES}

Athanasopoulos N \& Siakavellas N J (2017). Smart patterned surfaces with programmable thermal emissivity and their design through combinatorial strategies. Scientific reports, 7(1), 1-16.

Avdelidis N P \& Moropoulou A (2003). Emissivity considerations in building thermography. Energy and Buildings, 35(7), 663-667.

Biass S Orr T R, Houghton B F, Patrick M R, James M R \& Turner N (2019). Insights into pāhoehoe lava emplacement using visible and thermal structurefrom-motion photogrammetry. Journal of Geophysical Research: Solid Earth, 124(6), 56785695.

Candiago S, Remondino F, De Giglio M, Dubbini M \& Gattelli M (2015). Evaluating multispectral images and vegetation indices for precision farming applications from UAV images. Remote sensing, 7(4), 4026-4047. 
de Lima R S, Lang M, Burnside N G, Peciña M V, Arumäe T, Laarmann D, ... \& Sepp K (2021). An Evaluation of the Effects of UAS Flight Parameters on Digital Aerial Photogrammetry Processing and Dense-Cloud Production Quality in a Scots Pine Forest. Remote Sensing, 13(6), 1121.

Edelman G J \& Aalders M C (2018). Photogrammetry using visible, infrared, hyperspectral and thermal imaging of crime scenes. Forensic science international, 292, 181-189.

Erenoglu R C, Akcay 0 \& Erenoglu 0 (2017). An UASassisted multi-sensor approach for 3D modeling and reconstruction of cultural heritage site. Journal of cultural heritage, 26, 79-90.

Fraser C S (2013). Automatic camera calibration in close range photogrammetry. Photogrammetric Engineering \& Remote Sensing, 79(4), 381-388.

Guo Y, Senthilnath J, Wu W, Zhang X, Zeng Z \& Huang H (2019). Radiometric calibration for multispectral camera of different imaging conditions mounted on a UAV platform. Sustainability, 11(4), 978.

Matese A \& Di Gennaro S F (2018). Practical applications of a multisensor UAV platform based on multispectral, thermal and RGB high resolution images in precision viticulture. Agriculture, 8(7), 116.

Mello Román J C, Vázquez Noguera J L, Legal-Ayala H, Pinto-Roa D P, Gomez-Guerrero S \& García Torres M (2019). Entropy and contrast enhancement of infrared thermal images using the multiscale top-hat transform. Entropy, 21(3), 244.

Minařík R \& Langhammer J (2016). Use of a multıspectral uav photogrammetry for detection and tracking of forest disturbance dynamics. International Archives of the Photogrammetry, Remote Sensing \& Spatial Information Sciences, 41.

Murtiyoso A, Grussenmeyer P, Börlin N, Vandermeerschen J \& Freville T (2018). Open source and independent methods for bundle adjustment assessment in close-range UAV photogrammetry. Drones, 2(1), 3.

Nebiker S, Annen A, Scherrer M \& Oesch D (2008). A light-weight multispectral sensor for micro UAVOpportunities for very high resolution airborne remote sensing. Int. Arch. Photogramm. Remote Sens. Spat. Inf. Sci, 37(B1), 1193-1200.

Raeva P L, Šedina J \& Dlesk A (2019). Monitoring of crop fields using multispectral and thermal imagery from UAV. European Journal of Remote Sensing, 52(sup1), 192-201.

Ribeiro-Gomes K, Hernández-López D, Ortega J F, Ballesteros R, Poblete T \& Moreno M A (2017). Uncooled thermal camera calibration and optimization of the photogrammetry process for UAV applications in agriculture. Sensors, 17(10), 2173.
Sankey J B, Sankey T T, Li J, Ravi S, Wang G, Caster J \& Kasprak A (2021). Quantifying plant-soil-nutrient dynamics in rangelands: Fusion of UAV hyperspectral-LiDAR, UAV multispectralphotogrammetry, and ground-based LiDAR-digital photography in a shrub-encroached desert grassland. Remote Sensing of Environment, 253, 112223.

Saura J R, Reyes-Menendez A \& Palos-Sanchez P (2019). Mapping multispectral Digital Images using a Cloud Computing software: applications from UAV images. Heliyon, 5(2), e01277.

Turner R M, MacLaughlin M M \& Iverson S R (2020). Identifying and mapping potentially adverse discontinuities in underground excavations using thermal and multispectral UAV imagery. Engineering Geology, 266, 105470.

Van der Sluijs J, Kokelj S V, Fraser R H, Tunnicliffe J \& Lacelle D (2018). Permafrost terrain dynamics and infrastructure impacts revealed by UAV photogrammetry and thermal imaging. Remote Sensing, 10(11), 1734.

Wakeford Z E, Chmielewska M, Hole M J, Howell J A \& Jerram D A (2019). Combining thermal imaging with photogrammetry of an active volcano using UAV: an example from Stromboli, Italy. The Photogrammetric Record, 34(168), 445-466.

Wang J, Wang L, Jia M, He Z \& Bi L (2020). Construction and optimization method of the open-pit mine DEM based on the oblique photogrammetry generated DSM. Measurement, 152, 107322.

Webster C, Westoby M, Rutter N \& Jonas T (2018). Three-dimensional thermal characterization of forest canopies using UAV photogrammetry. Remote Sensing of Environment, 209, 835-847.

Wewel F, Scholten F \& Gwinner K (2000). High resolution stereo camera (HRSC)-multispectral 3Ddata acquisition and photogrammetric data processing. Canadian Journal of Remote Sensing, 26(5), 466-474.

Xu Z, Shen X, Cao L, Coops N C, Goodbody T R, Zhong T, ... \& Wu X (2020). Tree species classification using UAS-based digital aerial photogrammetry point clouds and multispectral imageries in subtropical natural forests. International Journal of Applied Earth Observation and Geoinformation, 92, 102173.

Zefri Y, ElKettani A, Sebari I \& Ait Lamallam S (2018). Thermal infrared and visual inspection of photovoltaic installations by UAV photogrammetry-application case: morocco. Drones, 2(4), 41.

Zumr D, David V, Jeřábek J, Noreika N \& Krása J (2020). Monitoring of the soil moisture regime of an earthfilled dam by means of electrical resistance tomography, close range photogrammetry, and thermal imaging. Environmental Earth Sciences, 79(12), 1-11. 